\title{
Experimental and Computational Analysis of the Unstable Flow Structure in a Centrifugal Compressor with a Vaneless Diffuser
}

\author{
Xiang Xue and Tong Wang *i]
}

\begin{abstract}
The unstable flow phenomena in compressors, such as stall and surge, are closely related to the efficiency and the operating region. It is indispensable to capture the unstable flow structure in compressors and understand the mechanism of flow instability at low flow rates. Cooperated with the manufacturer, an industrial centrifugal compressor with a vaneless diffuser is tested by its performance test rig and our multi-phase dynamic measurement system. Many dynamic pressure transducers are circumferentially mounted on the casing surface at seven radial locations, spanning the impeller region and the diffuser inlet region. The pressure fields from the design condition to surge are measured in details. Based on the multi-phase dynamic signals, the original location of stall occurring can be determined. Meanwhile, the information of the unstable flow structure is obtained, such as the circumferential mode and the propagating speed of stall cells. To get more details of the vortex structure, an unsteady simulation of this tested compressor is carried out. The computational result is well matched with the experimental result and further illustrates how the unstable flow structure in the impeller region gradually affects the stability of the total machine at low flow rates. The dynamic mode decomposition (DMD) method is applied to get the specific flow pattern corresponding to the stall frequency. Both experimental and computational analysis show that the flow structure at a particular radial location in the impeller region has a great impact on the stall and surge. Some differences between the computational and experimental result are also discussed. Through these two main analytical methods, an insight into the unstable flow structure in an industrial compressor is gained. The result also plays a crucial role in the guidance of the compressor stabilization techniques.
\end{abstract}

Keywords: Unstable flow, Vortex structure, Centrifugal compressor, Vaneless diffuser

\section{Introduction}

Unstable flow phenomena, like stall and surge, has always been a hot topic in the industry of compressors, but so far there are still many unresolved issues $[1,2]$. In the recent researches on centrifugal compressors, an insight into the internal unstable flow structure, especially at the tip clearance, played an important role in improving the stability of the overall machine [3-6]. The flow structure at the tip clearance is closely related to the stability of the compressor [7], thus some specific control methods [8, $9]$, such as casing treatment $[10,11]$, can greatly enlarge

\footnotetext{
*Correspondence: twang@sjtu.edu.cn

Gas Turbine Research Institute, Shanghai Jiao Tong University, Shanghai 200240, China
}

the stable operating range. In particular, according to some published experimental and computational results, the distorted pressure field in the tip region during rotating stall may greatly promote the flow instability, and even lead to surge [12-16].

In the experimental studies, a number of experiments were conducted on different centrifugal compressors by Lawless et al. [17]. In addition to the dynamic pressure and velocity transducers, the microphones were also used to record the characteristics during rotating stall and surge. In different experiments, the phenomenon that some special pressure fluctuation signals were propagated in the circumferential direction was always captured prior to stall dozens of revolutions. Due to the 
rotating stall, the circumferential pressure distribution at the specific radial position in the compressor is always in the form of high and low value alternating. Then, the pressure lobes rotate at a certain speed in the direction of the impeller rotating, resulting in a circumferential stall mode $[18,19]$. In the centrifugal compressor with a vaneless diffuser, the number of lobes is always two or three [12]. In recent years, a PIV experiment was carried out on a vaneless diffuser region of a centrifugal compressor by Ohuchida et al. [20], in which the velocity fields at different span height were obtained and the diffuser stall was analyzed qualitatively. Meanwhile, a 3-lobe stall structure can be clearly observed by the PIV experimental result. However, the number of the stall lobe was also found to be one recently in the vaneless diffuser of a centrifugal compressor [21]. The speed of this stall structure rotating is also the speed of the stall cell propagating circumferentially, which is generally considered between $5 \%$ and $30 \%$ of the impeller rotating speed [22]. These characteristics of the unstable flow structure have been valued by many researchers, so it is critical to accurately capture this unstable pattern and even to further analyze its relationship with stall and surge. In different measurement methods, the dynamic pressure signal is more stable than the speed or noise signal [23]. According to the multi-phase arrangement of pressure transducers, the key information about the propagation of stall cells, circumferential mode and return flow can all be obtained through the data post-processing.

In addition to experimental study, numerical simulation and theoretical analysis are also commonly used by researchers. Recently, Schleer et al. [24] carried out a simulation on the centrifugal compressor with vaneless diffuser and pointed out that the flow structure at impeller outlet was closely related to the tip leakage and vortex. Zhao et al. [4] further explored the relationship between the tip leakage flow and rotating stall through a numerical simulation of unstable flow fields in the Krain impeller. The result showed that there was a periodic pressure pulsation in the tip clearance at low flow rates, and the low frequency signals related to the tip leakage flow could be observed at the pre-stall condition. Compared with the experiment, numerical simulation can show a more comprehensive three-dimensional structure of flow fields. Thus, the extraction of flow structure characteristics from the computational result drew attentions of researchers. For example, Cizmas et al. [25] applied the Proper Orthogonal Decomposition (POD) method to a numerical simulation of the centrifugal compressor and successfully extracted the main flow structures of velocity and pressure fields. Schmid [26] proposed the dynamic mode decomposition (DMD) method based on the overall stability analysis. Through this method, the spatiotemporal evolution of different ordered structures in the unstable flow field can be obtained. Whether this analysis method can be applied to impeller machinery will also be explored in this paper.

In this research, a number of dynamic fast response pressure transducers are used in order to get sufficient unstable flow information. Based on the simultaneous acquisition of all dynamic pressure signals, the unstable flow characteristics at low flow rates, especially at the pre-stall condition, are all obtained. In order to further analyze the causes of the observed phenomena in the experiment, a two-passage numerical simulation and the DMD method are applied to the tested compressor as auxiliary analysis means. After verified by the experimental result, the computational result is used to provide data for the DMD method to extract the specific flow pattern corresponding to some dominating frequencies. Through the combination of the experiment and computational analysis, a gradual process is obtained that the tip leakage flow instability at a specific radial position of impeller causes a special stall mode at the impeller exit, leading to the diffuser stall and surge. An insight into the generation of this unstable flow structure in the centrifugal compressor is also gained.

\author{
1.1 Nomenclature \\ $b \quad$ Width $(\mathrm{mm})$ \\ $D \quad$ Diameter $(\mathrm{mm})$ \\ $f \quad$ Frequency $(\mathrm{Hz})$ \\ $Q \quad$ Flow rate $(\mathrm{kg} / \mathrm{s})$ \\ $T \quad$ Period (s) \\ $t$ Temperature (K) \\ $Z \quad$ Blade number \\ $\omega \quad$ Rotating speed $(\mathrm{rad} / \mathrm{s})$ \\ $\gamma \quad$ Specific heat ratio, $c_{\mathrm{p}} / c_{\mathrm{v}}$ \\ $\varepsilon \quad$ Pressure ratio, $P_{\text {out }} / P_{\text {in }}$ \\ $\eta \quad$ Adiabatic efficiency, $(\gamma-1) / \gamma \cdot \ln \left(p_{\text {out }} / p_{\text {in }}\right) /$ \\ $\ln \left(t_{\text {out }} / t_{\text {in }}\right)$
}
1.2 Subscripts
1 Impeller inlet
2 Impeller outlet
3 Diffuser inlet
$4 \quad$ Diffuser outlet
des Design condition
imp Impeller
in Inlet duct
max Maximum
out Outlet duct
$\mathrm{r}$ Impeller rotation 


\section{Experimental Facility and Instrument}

In order to understand the mechanism of unstable flow structures developing in the centrifugal compressor at small flow rates, especially at the pre-stall condition, the experimental study is carried out with the cooperation of manufacturer. An industrial centrifugal compressor with a vaneless diffuser is tested by its own performance test rig and our multi-phase dynamic measurement system.

\subsection{Performance Test Rig}

The experiments are based on an industrial centrifugal compressor with vaneless diffuser, which is always used in the sewage disposal system. The test rig for the experimental compressor is shown in Figure 1, where the including the sketch of the test rig and the corresponding photograph.

The compressor is driven by a $180 \mathrm{~kW}$ AC motor to rotate with speed of $18300 \mathrm{r} / \mathrm{min}$. The pressure ratio can reach the maximum of 1.88 at the design flow rate. Throttle valves are installed on the inlet and outlet pipes respectively. The flow rate is regulated by adjusting the outlet throttle and monitored through a Venturi flowmeter with relative error less than $0.5 \%$. Total pressures at the compressor inlet and outlet are recorded through

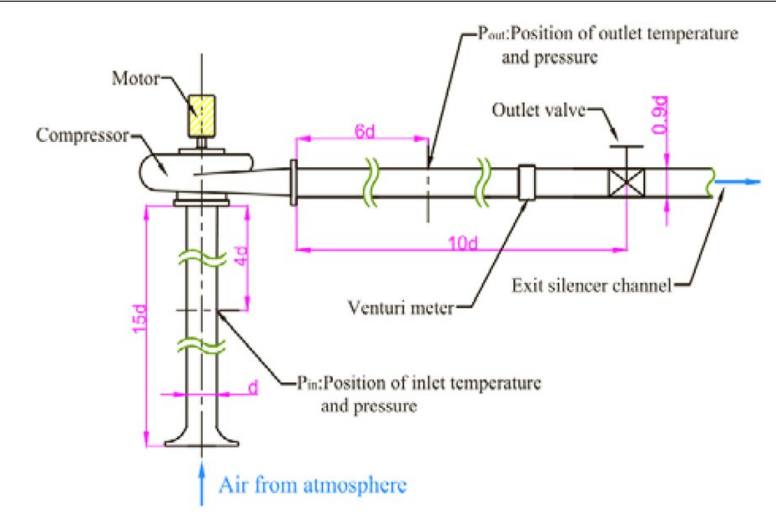

a Sketch of the test rig

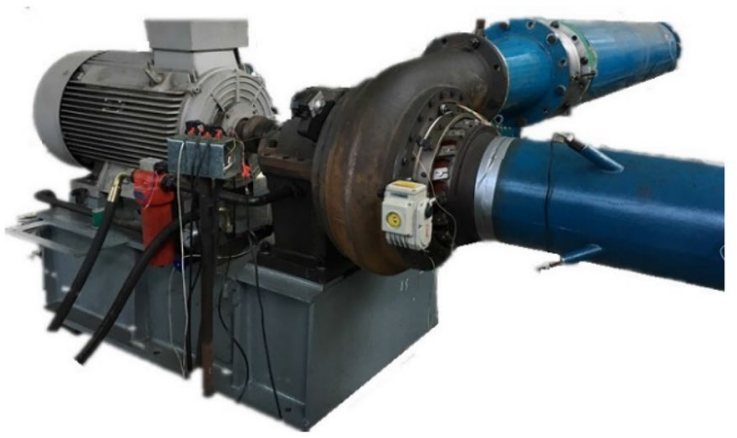

b Photograph of the test rig

Figure 1 Test rig for the experimental compressor
Pitot tubes with relative error less than $0.26 \%$. Temperatures at the compressor inlet and outlet are recorded through thermocouples with relative error less than $0.25 \%$. The specific parameters of the test compressor are shown in Table 1.

\subsection{Multi-Phase Dynamic Measurement System}

The multi-phase dynamic pressure test system can be seen in Figure 2. A number of dynamic pressure transducers are arranged at seven different radial positions, spanning the impeller region and diffuser inlet region in the test compressor. The distribution of these dynamic transducers is shown in Figure 2(a). At each radial position, multi-phase transducers are circumferentially

Table 1 Parameters of the test compressor

\begin{tabular}{llllll}
\hline Parameter & Value & Unit & Parameter & Value & Unit \\
\hline$D_{1}$ & 89 & $\mathrm{~mm}$ & $D_{3}$ & 170 & $\mathrm{~mm}$ \\
$D_{2}$ & 160 & $\mathrm{~mm}$ & $D_{4}$ & 265 & $\mathrm{~mm}$ \\
$b_{2}$ & 24 & $\mathrm{~mm}$ & $Q_{\text {des }}$ & 2.59 & $\mathrm{~kg} / \mathrm{s}$ \\
$Z_{\text {imp }}$ & $8+8$ & & $\varepsilon_{\max }$ & 1.88 & \\
\hline
\end{tabular}

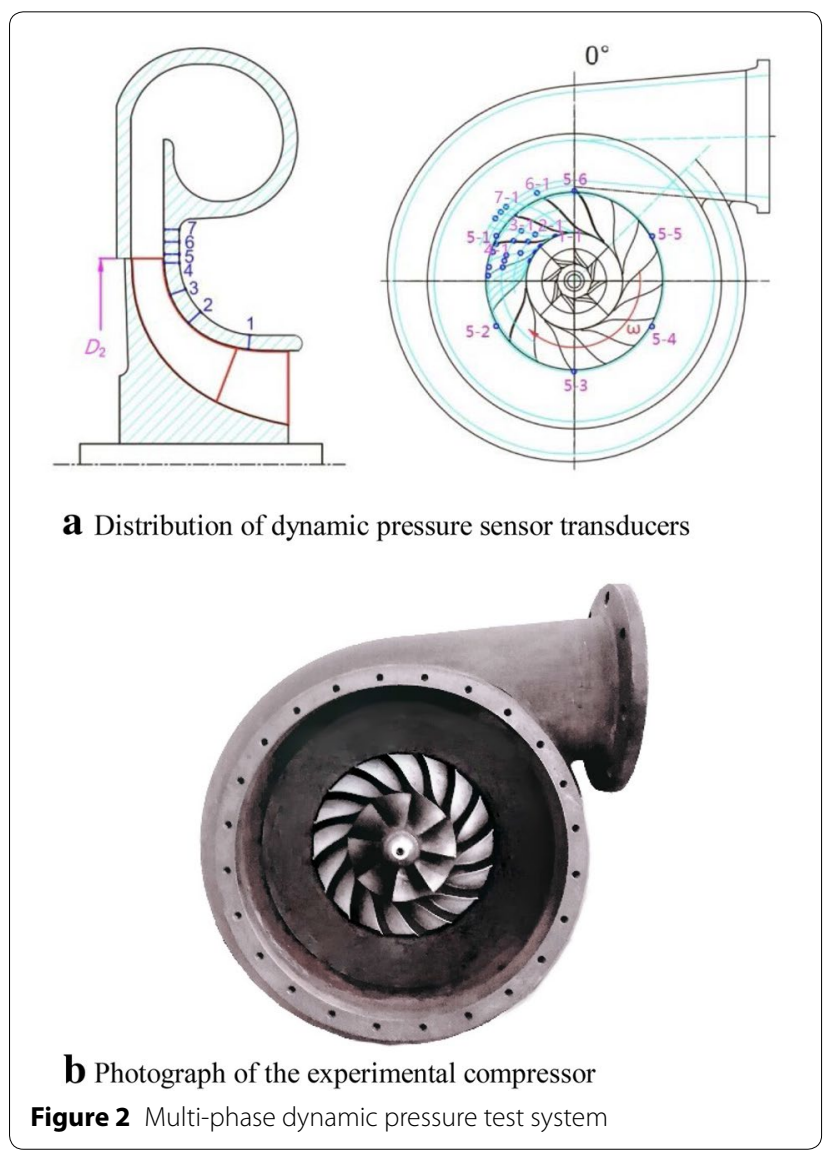


arranged. Corresponding to the circumferential view of the transducer distribution in the right of Figure 2(a), the photograph of the experimental compressor is presented in Figure 2(b), including a centrifugal impeller, a vaneless diffuser and an asymmetric volute. These transducers are embedded in the casing surface and the probes are flush with the inner wall. The real-time signals at different positions are recorded synchronously by a signal acquisition device, thereby dynamic pressure fields near the tip clearance at different operating conditions can be gained.

The model of pressure transducers is Kulite XCE093 belong to piezoresistive sensor with measuring accuracy of $0.05 \%$, whose probe diameter is $2.4 \mathrm{~mm}$. The measuring range of all these transducers is 0 to $300 \mathrm{kPa}$. The signal acquisition relies on eight NI 9215 acquisition cards and a DAQ-9178 chassis. The total relative measuring error of signal acquisition device and transducers is less than $0.5 \%$. In order to verify the accuracy of the dynamic pressure measurement, the blade passing frequency $(\mathrm{BPF}=2440,4880 \mathrm{~Hz})$ need be captured, so the sampling frequency for each channel was set as $20 \mathrm{kHz}$.

In the dynamic test, through the gradual closure of the outlet valve, the operating state of this test compressor is adjusted from the design point to surge. In each step of this gradual process, the pressure data for all measuring points are collected in real time, especially when pre-stall, stall and surge. These large amounts of data are carefully stored in the computer, which are important bases for the analysis of flow instability, such as the stall mode.

\section{Experimental Results and Discussion}

Through the performance test, the approximate location of stall and surge can be determined. According to the real-time signal analysis under small flow rates, the dynamic test is mainly concerned with the characteristics of the unstable flow structure.

\subsection{Performance Test Results}

The performance test result of the compressor is represented by solid dots and lines in Figure 3, including the total pressure ratio curve $(\varepsilon)$ and the adiabatic efficiency curve $(\eta)$. Their expressions have been demonstrated in the Section 1.1. Figure 3 illustrates that the adiabatic efficiency begins to decrease since the design point (I) to the left, while the pressure ratio continues to rise until the stall inception point (III) where the modal stall inceptions can be observed in the real-time signals. Moreover, the small flow rate test region is the focus of the dynamic test, from the originating point (II) of the stall disturbance to the surge point (IV).

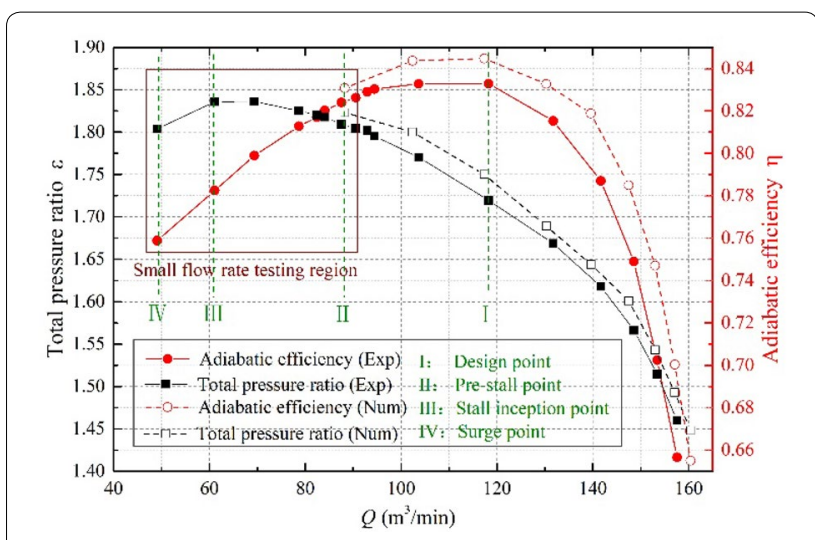

Figure 3 Performance map of the test compressor

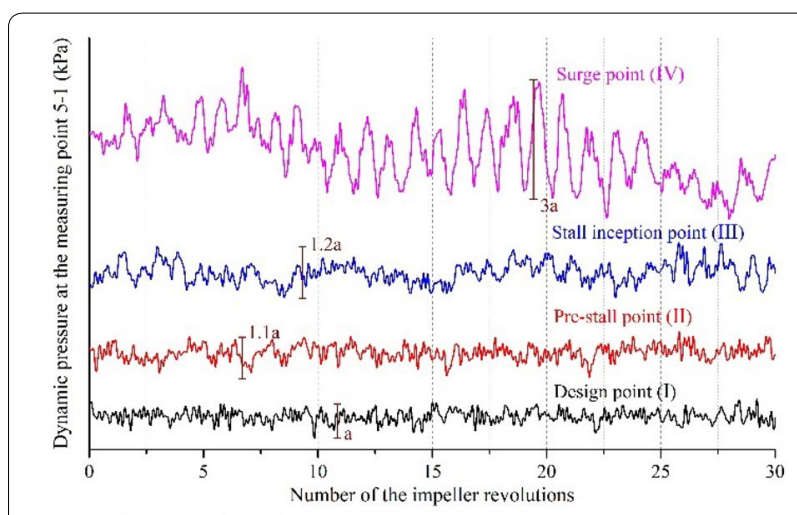

a In the time domain

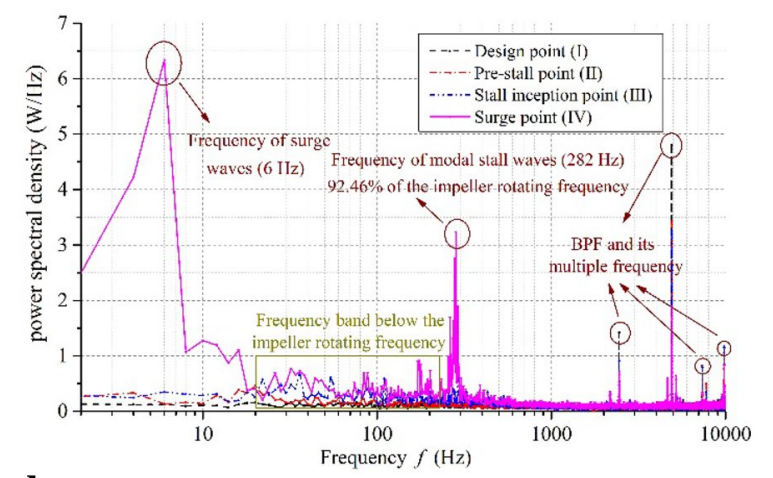

b In the frequency domain

Figure 4 Comparison of pressure signals at different conditions

\subsection{Dynamic Pressure Test Results}

Focusing on the measuring point 5-1 as an example, the comparison among the dynamic pressure signals at different operating conditions is shown in Figure 4(a). The dynamic pressure curves are arranged in order from bottom to top, independent of the absolute value. The absolute value of dynamic pressure is ignored since it is not the focus of this research. However, both the ordinate 
unit and scale of all the curves are consistent. Thus, the magnitudes of various signal fluctuations in Figure 4(a) can be compared. It can be seen that the pressure fluctuation is more stable at the design condition while the fluctuating amplitude and the flow instability increases since the pre-stall point. Until the surge point, the obvious modal stall waves and surge waves appear, with the pressure fluctuating amplitude greatly increasing.

Their respective spectrum analyses are shown in Figure 4(b). The BPF and its multiplier are fixed in different cases. At the design condition, the energy amplitudes of the frequency band less than the impeller rotating frequency are all basically below 0.1, without any significant peaks. While since the pre-stall point, many small peaks appeared within this frequency region, and the overall energy amplitude increased gradually. Since this frequency region is related to the flow instability [12], the amplitude rise of the frequency components in this region indicates that the internal flow deteriorates. Until the surge point, in the low frequency region, the frequency component of surge waves $\left(f_{\text {surge }}=6 \mathrm{~Hz}\right)$ with the energy amplitude of 6 and the frequency component of modal stall waves $\left(f_{\text {stall }}=282 \mathrm{~Hz}\right)$ with the energy amplitude of 3 stand out one after another. In addition, at the small flow rate conditions, there are some small peaks appearing in the vicinity of BPF.

In order to further capture the originating position of the stall inception, the dynamic pressure signals at different radial positions under the stall inception condition (III) are compared, as shown in Figure 5. The stall wave originates at the impeller middle-rear region (the radial third row of measuring points) when there is no significant stall wave signal at other radial positions.

In the repeated experiments, the impeller middle-rear region is all the originating position of the modal stall inception, which shows that the tip leakage flow and vortex in the impeller region is the main factor of stall with the

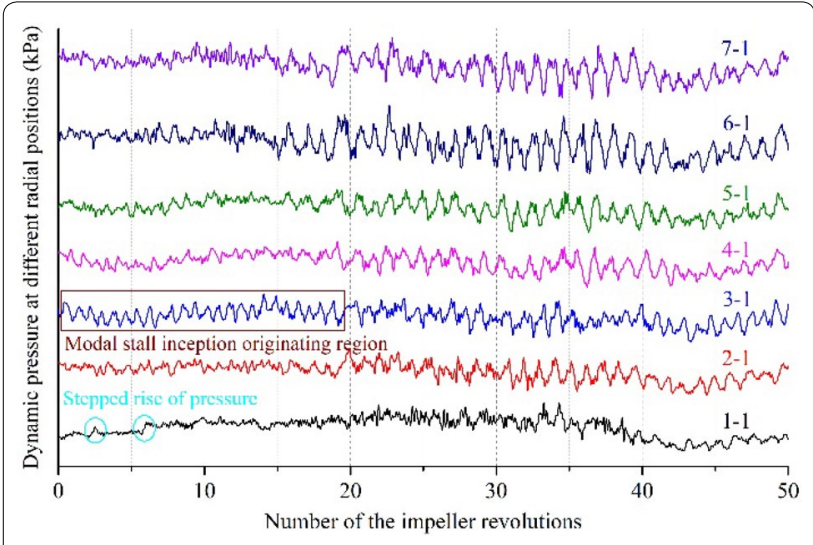

Figure 5 Modal stall inceptions at different radial positions overall pressure rising ability and the flow rate reducing. Then, the stall inception occurred at each radial position, and soon the compressor suffered a surge. At this moment, a huge periodic noise caused by the reflux and duct vibration can be clearly heard. In addition, there are pressure stepped rising phenomena at the impeller inlet (the radial first row of measurement points) occurring prior 20 revolutions to the occurrence of the stall inception.

\subsection{Discussion of Unstable Flow Patterns}

At the surge condition (IV), the alternate mode of stall and surge occurs at each position in the compressor, and the signals of the circumferential six measuring points at the impeller outlet are shown as an example shown in Figure 6(a). In this case, there is a low-frequency fluctuation in the dynamic pressure signal with the period of about 40 revolutions. Its frequency is close to the fluctuating frequency of the surge wave in Figure 4(b), so it can be regarded as the surge frequency. In this low-frequency fluctuation period, a relatively high-frequency pressure fluctuation generates intermittently with a frequency of about $90 \%$ of the blade rotating frequency, which

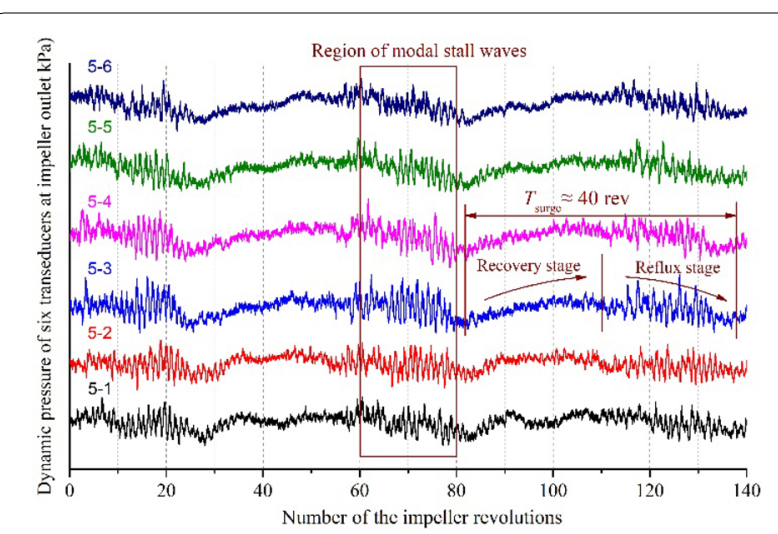

a Signals of surge waves

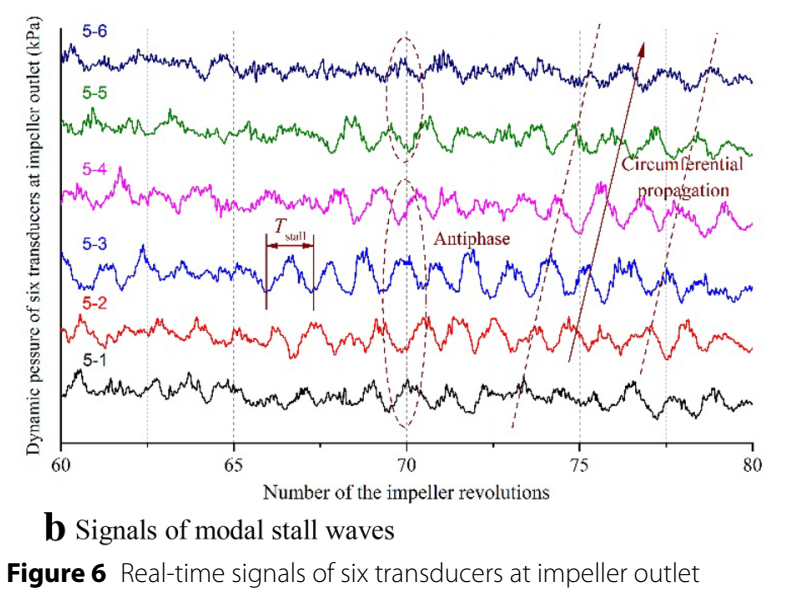


can be considered as the frequency of the modal stall in Figure 4(b).

In general, the stall evolves into a mutant type after a full development. The flow deteriorates and then the surge occurs. However, this mode of stall and surge alternating belongs to a progressive stall type, where stall and surge often occur in turn. This mode has been found in the experiments conducted by other researchers in recent years [23, 27]. It suggests that the compressor is transitioned from stall to surge gradually rather than abruptly. A surge cycle is divided into two stages, which are the reflux stage and the recovery stage. In the reflux stage, since the compressor suffers a surge, the flow instability is exacerbated, and then the impeller rotation can no longer make the compressor achieve the corresponding pressure ratio. The pressure at the compressor outlet is reduced while the back pressure in the outlet duct is still maintained at the previous value due to the inertia, which makes the air flow reflux occur because of the pressure difference. And there is a clear modal stall phenomenon in the reflow process. While, in the recovery stage, due to the occurrence of the reflux, the back pressure in the outlet duct is reduced to the pressure at the compressor outlet. The internal flow begins to recover.

Figure 6(b) is a partial amplification of the modal stall wave region in Figure 6(a). An interesting phenomenon can be found that the phases of the stall wave signals at any adjacent two measuring points at the impeller outlet are all almost reverse. The six points at the impeller outlet are evenly distributed along the entire circumference, with any two adjacent points separated by $60^{\circ}$. Thus, the stall form at this time is an approximately axisymmetric three-lobe stall pattern, as shown in Figure 7. It is also generally believed that the number of stall lobes in a centrifugal compressor with vaneless diffuser was two or three [12]. Three stall cells rotate along the same direction with the same rotating speed, so as to ensure that the signal phases of any two adjacent points at the impeller outlet were almost reverse. Since the number of the stall mode is three, the actual rotating frequency of stall waves is $282 / 3=94 \mathrm{~Hz}$, and the propagating speed $\left(\omega_{\text {stall }}\right)$ is $30.82 \%$ of the impeller rotating speed.

In this experiment, based on the real-time signals of the six circumferential measuring points at the impeller outlet, both the modal number and the circumferential propagating speed of stall were obtained. Meanwhile, according to the signals of different radial measurement points at the stall inception condition, the stall originated at the impeller middle-rear region, resulting in a comprehensive stall and surge. However, some researchers, through the experiments and simulations, suggested that the vaneless diffuser always played a more important role

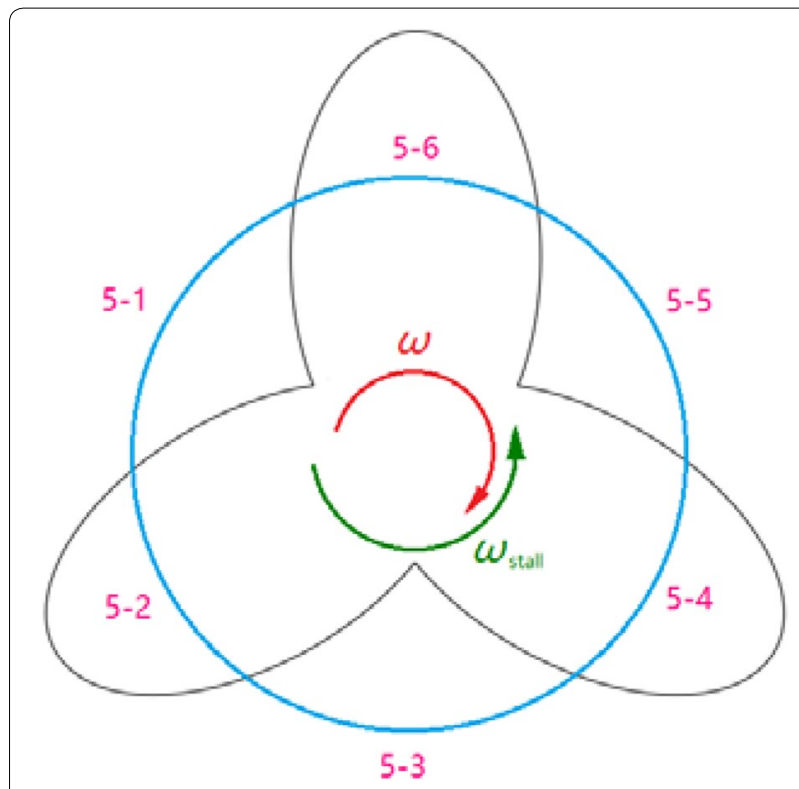

Figure 7 Schematic diagram of the three-lobe stall pattern

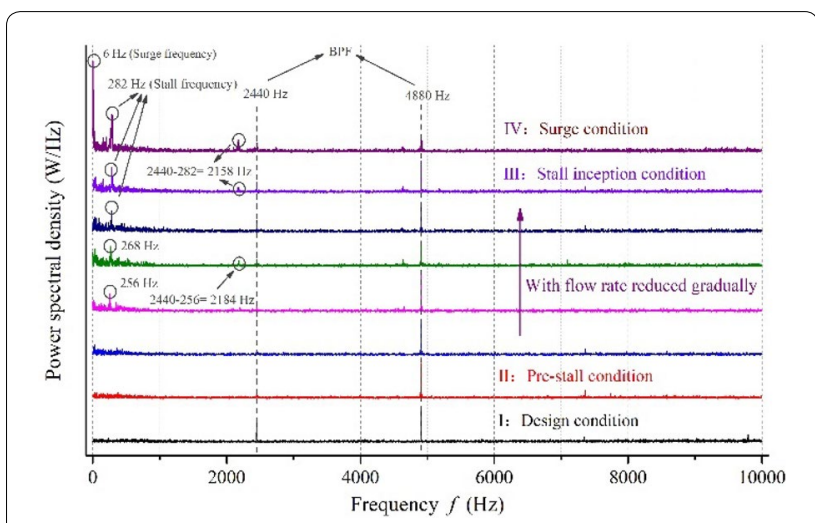

Figure 8 Spectrum analysis in the diffuser at different conditions

in the overall instability process than the impeller [12, 20]. Also, many researchers began to focus on the effects of tip leakage vortex and flow in the impeller on the overall instabilities of centrifugal compressors [4, 28].

In order to further explore the characteristics of the diffuser stall, the spectral analysis of the signals in the diffuser under different conditions is carried out, as shown in Figure 8 . In contrast to the situation in the impeller, from the pre-stall state to the stall state, a frequency component of the stall mode with a small energy peak occurred in the diffuser before that in the impeller. However, according to Figure 5, at the stall inception condition, the obvious modal stall wave did originate at the impeller middle-rear region. There was a regular phenomenon that since the occurrence of the stall mode frequency in the diffuser, this 
frequency gradually increased with the flow rate reducing, until the stall inception condition. It is probable illustrated that, due to the asymmetrical distribution of pressure field at the impeller outlet, the prototype of the three-lobe stall mode was gradually formed in the diffuser and its propagation was accelerated with the flow rate reducing. And the leakage flow and vortex at the impeller middle-rear region made the instability of this stall mode increase, triggering a large-scale stall and surge.

\section{Computational and Theoretical Analysis}

Based on the experimental analysis, the characteristics of the unstable flow, such as the form and the initial position of stall, are obtained. However, the most concerned issue about the inertial flow details, such as how the tip leakage and separation vortex in the impeller gradually affects the diffuser region or even the overall machine, has not been intuitively displayed. In order to analyze the causes of the observed phenomena in the experiments, a two-passage numerical simulation and DMD method are applied to the tested compressor as auxiliary analysis means.

\subsection{Numerical Model}

The complete full-circumferential model and the selected computational region are shown in Figure 9. The complete physical model in Figure 9(a) includes a centrifugal impeller and a vaneless diffuser. All the dimensions are referenced to the tested compressor, consistent with Table 1 . The gas is drawn from the impeller inlet and then removed from the diffuser outlet. Ignoring the effects of the inlet duct and the volute, this is a simple model of a centrifugal compressor, to study the flow instability in the impeller and the diffuser. In addition, since this model is axisymmetric, its circumferential part is selected for numerical calculation in order to save computational cost and time. The selected calculation region and the corresponding computational grid are shown in Figure 9(b). Two channels of the tested impeller, including two main vane channels and two guide vane channels, with the corresponding vaneless diffuser region are selected as the numerical model. This simplified model can be used to simulate the complete full-circumferential model by setting the periodic boundary condition.

\subsection{Parameter Settings and Boundary Conditions}

The commercial software ANSYS CFX is used for the numerical calculations. The governing equations for the numerical simulation are the Reynolds averaged NavierStokes equations, as follows:

$$
\left\{\begin{array}{l}
\frac{\partial\left\langle u_{i}\right\rangle}{\partial t}+\left\langle u_{j}\right) \frac{\partial\left\langle u_{i}\right\rangle}{\partial x_{j}}=-\frac{1}{\rho} \frac{\partial\langle p\rangle}{\partial x_{i}}+v \frac{\partial^{2}\left\langle u_{i}\right\rangle}{\partial x_{j} \partial x_{j}}-\frac{\partial\left\langle u_{i}^{\prime} u_{j}^{\prime}\right\rangle}{\partial x_{j}}, \\
\frac{\left.\partial u_{i}\right\rangle}{\partial x_{i}}=0 .
\end{array}\right.
$$

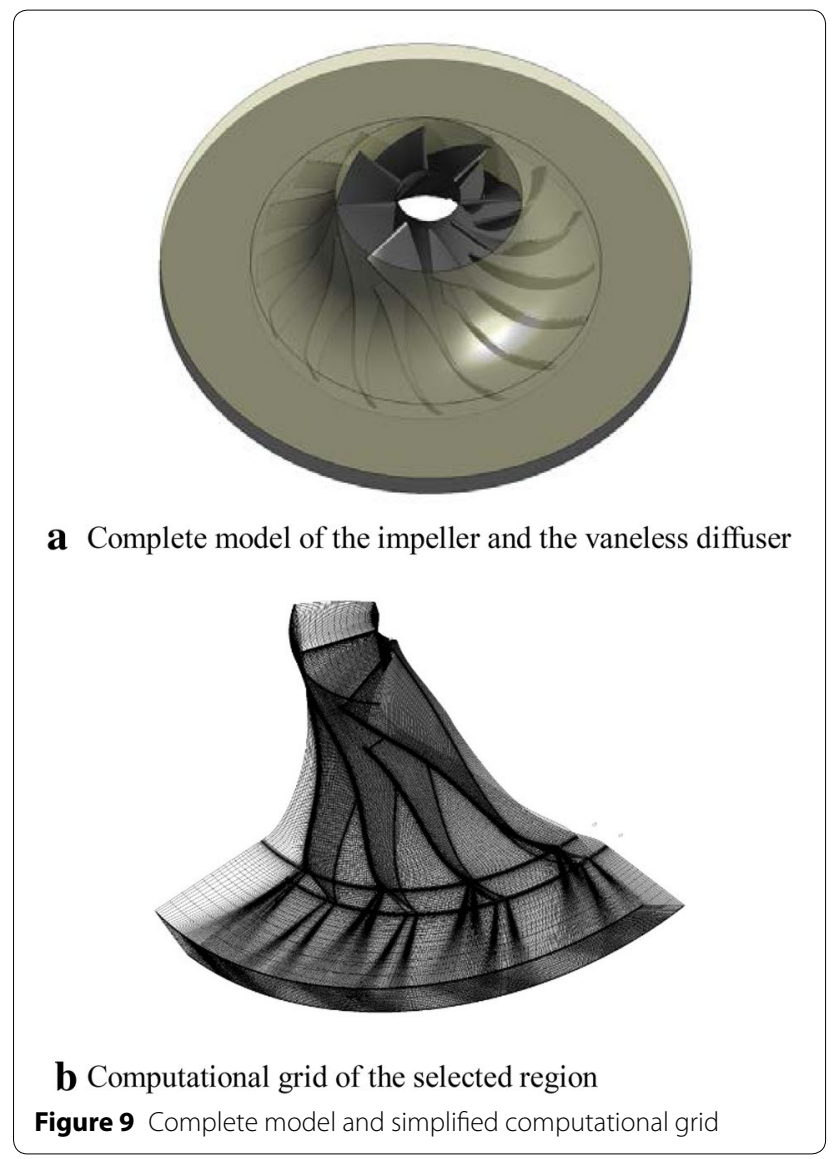

In the numerical simulation, the boundary conditions are set according to the performance map shown in Figure 3. The axial uniform inflow condition is given at the compressor inlet, with a total pressure of $101325 \mathrm{~Pa}$ and a total temperature of $300 \mathrm{~K}$. An average static pressure condition is set at the compressor outlet. The adiabatic solid wall condition is applied to the compressor surface. The rotational periodic boundary conditions are imposed on the impeller channels. The ideal air model is used for the internal fluid, and the viscous effect is taken into account. In order to better capture the vortex structure near the shroud side of the test compressor, the SST $k-\omega$ model is chosen as the turbulence model. As a two-equation model, it assumes the relationship between the mean and instantaneous values of each parameter in the flow field, thereby closing the time-averaged Navier-Stokes equations. In the calculation, the high-precision format is selected in the spatial domain, and the second-order difference format is selected in the time domain.

The grid of the computational model is generated by the commercial software Turbogrid, which is shown in Figure 9. H-type grid is used in the mainstream area 


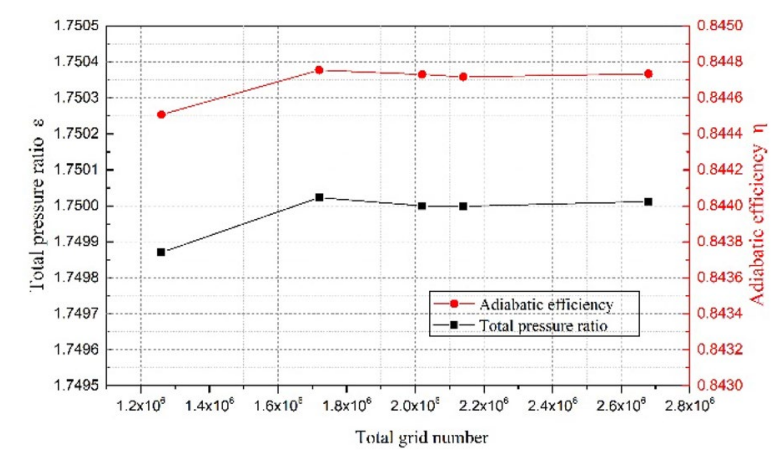

Figure 10 Grid independence analysis

and O-type grid is used in the vanes around, in order to ensure the quality of the grid. The smallest angle between the grid faces is $17.36^{\circ}$, and the wall grid dimensionless coefficient $y^{+}$is less than 2.5 , which all meet the requirements of the numerical simulation. The grid independence analysis is shown in Figure 10. When the total gird number is more than two million, both the adiabatic efficiency $(\eta)$ and total pressure ratio $(\varepsilon)$ of the two-passage numerical calculation at the design condition basically remain constant. Thus, the total grid number of the computational model is chosen as 2.02 million.

The calculation process is divided into two phases:

(1) In the first stage, the Frozen stator condition is set at the impeller inlet and outlet. According to the given boundary conditions, a steady calculation is carried out.

(2) In the second stage, the solution converged in the first stage is treated as an initial flow field for a further unsteady calculation. The frozen stator condition is converted into the Transient Rotor/Stator condition.

Through the adjustment of the static pressure at the vaneless diffuser outlet, the flow fields at different operating conditions can be obtained. In the unsteady calculation, the time step is taken as $1.2807377 \times 10^{-5} \mathrm{~s}$, that is, the impeller need spend 256 steps in rotating a circle.

\subsection{Validation of the Numerical Simulation}

As shown in Figure 3, both the total pressure ratio curves and the adiabatic efficiency curves of the two-passage unsteady numerical calculation and the experiment are compared respectively. In Figure 3, experimental operating points are marked with solid points, while computational operating points are marked with hollow points. Their trends are consistent. Especially from the design condition (I) to the pre-stall condition (II), these two adiabatic efficiencies both begin reducing from the highest, while the pressure ratios both keep growing.

However, at the same flow rate, both the pressure ratios and efficiencies of the computational operating points are higher than those of the experiment. The relative error of efficiency is less than $3.9 \%$, and that of pressure ratio is less than $1.6 \%$. Since the computational model does not include the volute and the outlet pipe, part of the flow loss has not been calculated, leading to such a small deviation. Thus, the performance results of the numerical simulation can be considered reasonable, and the internal flow field data can be used to analyze the flowing characteristics.

\subsection{Dynamic Mode Decomposition (DMD) Method}

Compared with the proper orthogonal decomposition (POD) method, the dynamic mode decomposition (DMD) method is a more advanced modal decomposition method, which is originated from the Koopman operator in the overall stability analysis. Through the Koopman mapping, flow fields could be expanded into various modes of superposition. The Koopman operator is often used to analyze nonlinear dynamical systems. In theory, all the information of a nonlinear dynamical system can be captured through the infinite dimension of the Koopman operator [29].

For example, the flow field data sampled with same time intervals are represented by a matrix as follows:

$$
\boldsymbol{V}_{1}^{n}=\left\{\boldsymbol{v}_{1}, \boldsymbol{v}_{2}, \cdots \boldsymbol{v}_{n}\right\} .
$$

Then, assuming that there is a linear mapping $\boldsymbol{A}$ to meet this condition in Eq. (3), this matrix can be considered as a system matrix reflecting the spatiotemporal evolution of the flow field:

$$
\boldsymbol{v}_{i+1}=A \boldsymbol{v}_{i+1} .
$$

Based on the Arnoldi algorithm, the eigenvalues of this matrix are solved iteratively. Eq. (2) is transformed into Eq. (3) in mathematical form:

$$
V_{2}^{n}=A V_{1}^{n-1} \approx V_{1}^{n-1} S,
$$

where the adjoint matrix $S$ reflects the eigenvalue information of the system matrix $A$. The key to this algorithm is to find a suitable adjoint matrix $S$ to minimize the residual of Eq. (3). The matrix $V_{1}^{n-1}$ is decomposed by the singular value decomposition (SVD):

$$
\boldsymbol{V}_{1}^{n-1}=\boldsymbol{U} \Sigma \boldsymbol{V}^{\mathrm{T}}
$$

A matrix $\widetilde{S}$ is presented as follows:

$$
\widetilde{\boldsymbol{S}} \equiv \boldsymbol{U}^{\mathrm{T}} \boldsymbol{A} \boldsymbol{U}=\boldsymbol{U}^{\mathrm{T}} \boldsymbol{A}\left(\boldsymbol{U} \Sigma \boldsymbol{V}^{\mathrm{T}}\right) \boldsymbol{V} \Sigma^{-1}=\boldsymbol{U}^{\mathrm{T}} \boldsymbol{V}_{2}^{\mathrm{n}} \boldsymbol{V} \Sigma^{-1} .
$$

The eigenvalues of the matrix $\widetilde{S}$ are equivalent to the eigenvalues of the matrix $\boldsymbol{A}$, so the eigenvalues $\lambda_{i}$ and 
eigenvectors $\boldsymbol{y}_{i}$ of the system matrix $\boldsymbol{A}$ can be written as follows:

$$
\widetilde{\boldsymbol{S}} \boldsymbol{y}_{i}=\lambda_{i} \boldsymbol{y}_{i}
$$

Finally, the dynamic mode decomposition can be expressed as follows:

$$
\boldsymbol{\Phi}_{i}=\boldsymbol{U} \boldsymbol{y}_{i} .
$$

The eigenvalues $\lambda_{i}$ of modals $\Phi_{i}$ can be represented by complex planes. In the complex plane, the modal flow is relatively certain with an eigenvalue on the unit circle, while it is random with an eigenvalue deviating from the unit circle. The modal energy of flow fields can be expressed by the norm $\left|\Phi_{i}\right|$ of the corresponding modals. The steady modals with high energy are selected for the flow field analysis, while those with low energy reflects the information of the original flow field.

\subsection{Theoretical Analysis of the Numerical Results}

The computational performance lines are represented by hollow dots and dashed lines, as shown in Figure 3. The flow rate is decreased until the pre-stall point (II). From the pre-stall point, if the flow is reduced again, the unsteady calculation will be significantly divergent. Thus, the pre-stall point is the minimum flow rate point that can be achieved in this unsteady calculation. The calculation runs at this point for about 20 impeller revolutions, until the pressure signals in the compressor fluctuates slightly. Then, the result in the last few revolutions is extracted for detailed study.

At the design condition (I) and the pre-stall condition (II), the results at the final calculation steps of the unsteady simulation are selected for comparison. The final steps are both taken at the 20th revolution after convergence. The different transient streamlines in the impeller channels are shown in Figure 11, where the red lines represent the tip leakage streamlines and the black lines represent the main streamlines. In addition, the flow field on the tip clearance cross section $\left(b / b_{2}=0.9\right)$ at the impeller outlet is represented by the distribution of the relative Mach number. At the design condition (I), as shown in Figure 11(a), there is no obvious flow separation phenomenon at the leading edge of the main vanes. Most of the fluid is imported from the inlet into the corresponding channel while a small amount of the fluid with low Mach numbers becomes the tip leakage flow at the impeller middle-rear region. Combined with the streamlines and velocity distributions, it can be concluded that there is a three-dimensional vortex structure at the impeller channel, caused by the leakage flow at the impeller middle-rear region. When the flow rate is reduced to the pre-stall condition (II), it can be seen from Figure 11(b) that there is a clear flow separation phenomenon on the suction surface

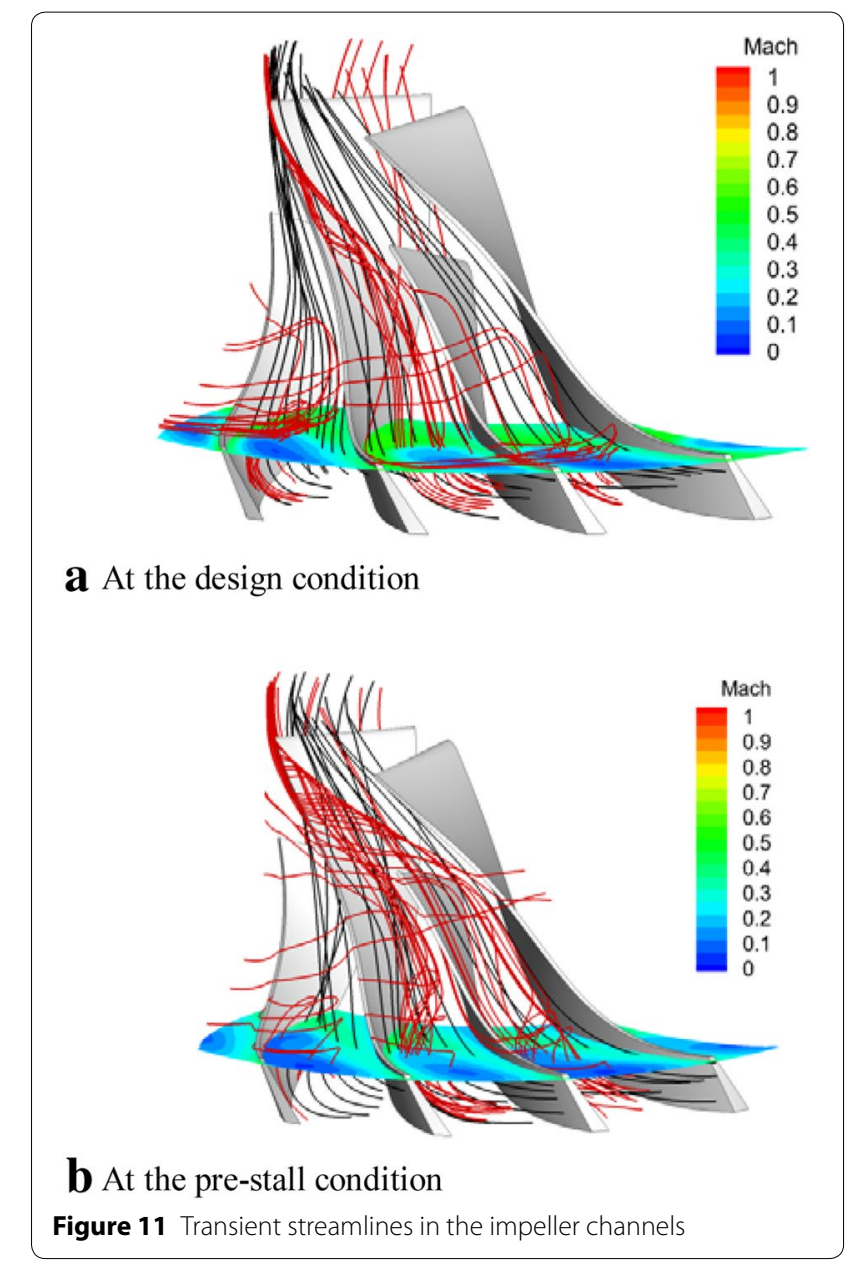

at the leading edge of the main vanes, which is evolving into the tip leakage flow. As a result, the flow at the impeller middle-rear region is worsened and the low-speed areas in the tip region at the impeller outlet move toward the suction surface of the main vanes. In the experiments, the modal stall inceptions originate at the impeller middle-rear region. Thus, it can be further determined that the flow instability at the impeller middle-rear region is co-excited by the tip leakage flow and the flow separation at the impeller leading edge.

In the next step, the DMD method is applied to the flow fields at the pre-stall condition, in order to extract the specific flow patterns of different frequency segments and analyze their stability. In the unsteady simulation, the sampling frequency is $19520 \mathrm{~Hz}$, which is close to the sampling frequency of $20 \mathrm{kHz}$ in the experiment. The flow field data during twice the impeller rotating periods are subjected to DMD processing. In theory, with this sampling frequency and time, the flow patterns corresponding to the spectrum from $150 \mathrm{~Hz}$ to $9760 \mathrm{~Hz}$ can be captured by the DMD algorithm. The modal 

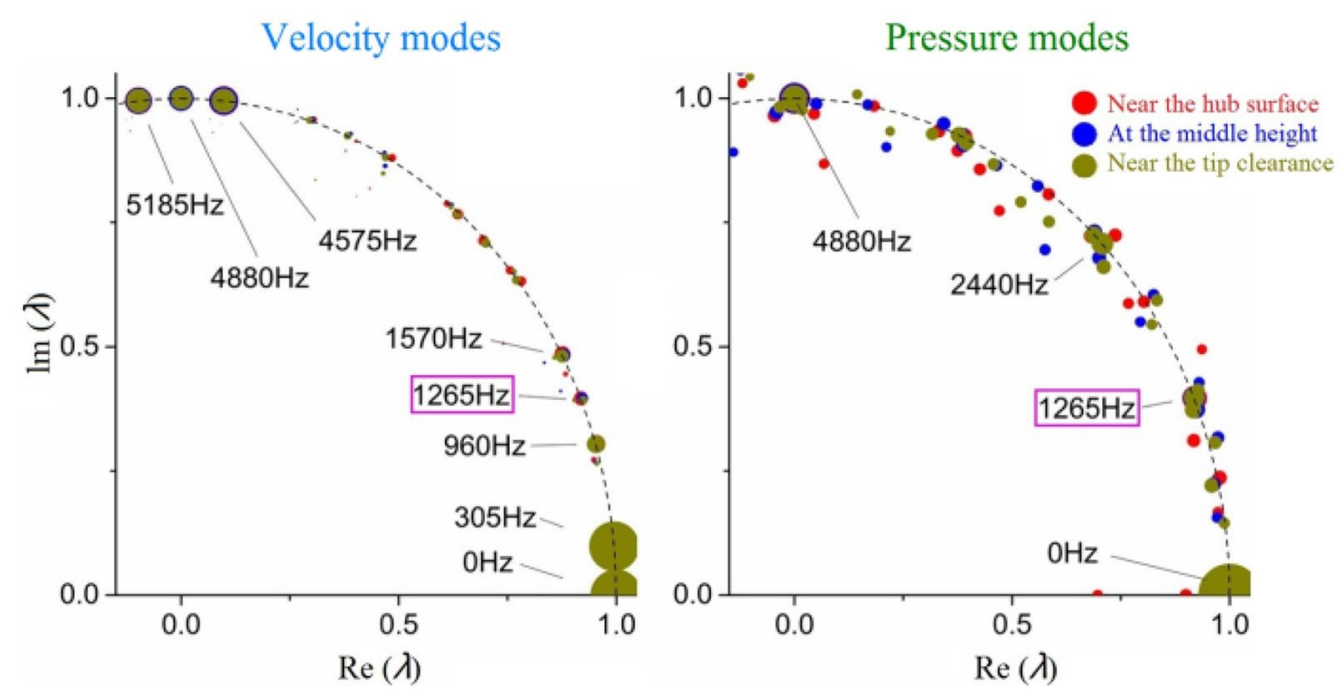

\section{a At the impeller outlet}
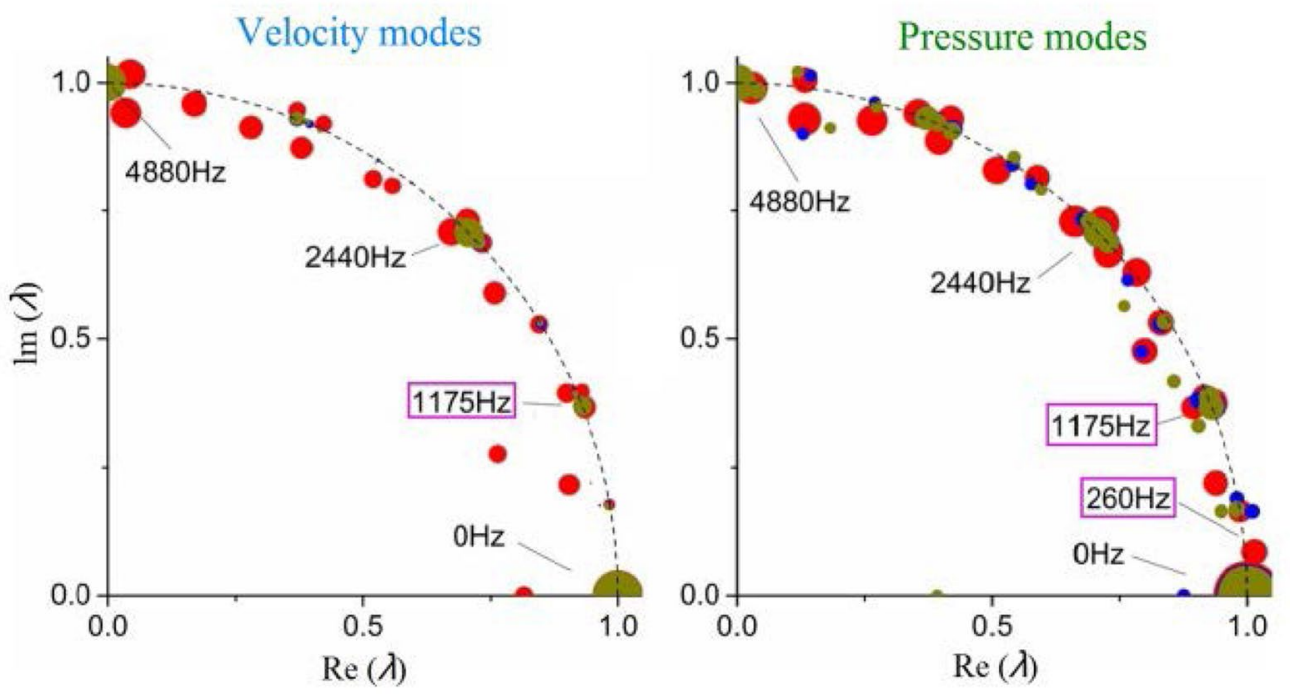

\section{b In the vaneless diffuser}

Figure 12 Modal decomposition spectrums of the flow fields

decomposition spectrums of the velocity and pressure fields at different height sections of the impeller outlet and vaneless diffuser are shown in Figure 12, where the abscissa is the real part of the eigenvalue $\lambda$, and the ordinate is the imaginary part of the eigenvalue $\lambda$. The dots correspond to modal frequencies with different frequencies, and the size of the dots correspond to their modal energy. The three different colors of these dots represent the different height positions of the corresponding modals. These are all obtained through the DMD method. The dot on the abscissa corresponds to the frequency component of 0 , which represents the time-independent average information in the flow field.

In these modal spectrums, the components corresponding to BPF are significant. And there is a modal component corresponding to the impeller rotating frequency $\left(f_{\mathrm{r}}=305 \mathrm{~Hz}\right)$ in the modes of the velocity field rather than the pressure field. It explains why the impeller 

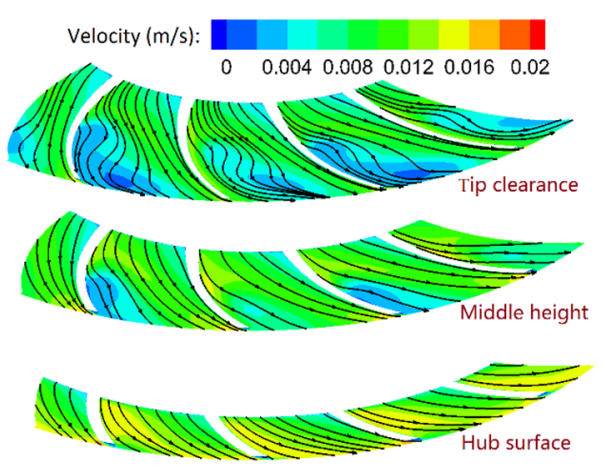

a Velocity field of $f=305 \mathrm{~Hz}$ at the impeller outlet

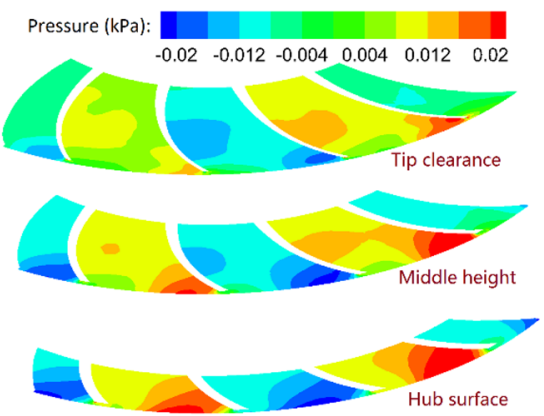

C Pressure field of $f=1265 \mathrm{~Hz}$ at the impeller outlet

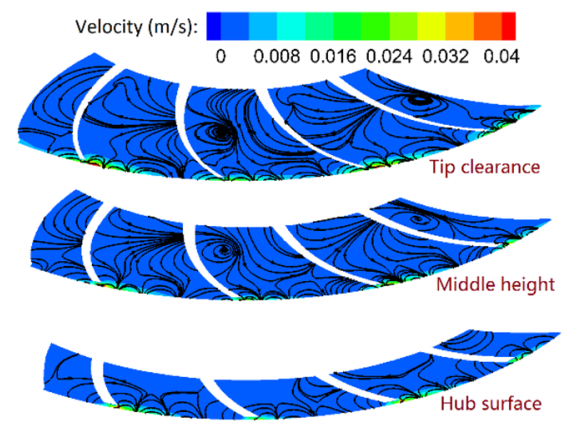

b Velocity field of $f=4880 \mathrm{~Hz}$ at the impeller outlet
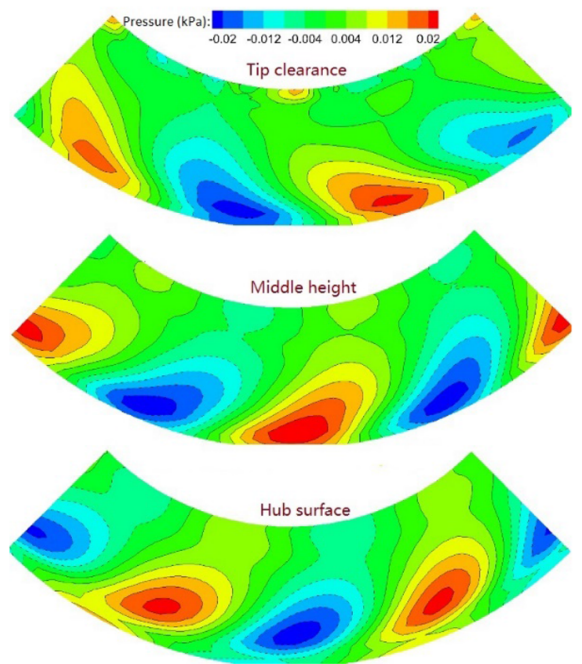

d Pressure field of $f=1175 \mathrm{~Hz}$ in the vaneless diffuser

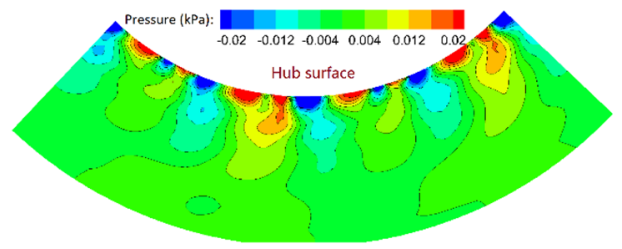

C Pressure field of $f=260 \mathrm{~Hz}$ in the vaneless diffuser

Figure 13 Flow fields of the dominating modals

rotating frequency is not found in the experimental pressure signal spectrum, while the BPF is obvious. In addition to the components of $\mathrm{BPF}$ and $f_{\mathrm{r}}$, there are many frequency components deviating from the unit circle in the modal decomposition spectrum. According to the DMD theory, these frequency components are all random patterns at this time. As described in Figures 5 and 8 , there is no clear stall frequency component from the design point to the pre-stall point, but some other frequency components produced and disappeared randomly, which are likely to be some uncertain vortices.

Focusing on the frequency components on the unit circle, some noteworthy frequencies can be found, which are marked in Figure 12. First, the component with $f_{\mathrm{r}}=305 \mathrm{~Hz}$ that appeared only in the velocity modal spectrum at the impeller outlet is shown in Figure 13(a). It can be seen that the streamlines of the modal with $f_{\mathrm{r}}=305 \mathrm{~Hz}$ describes the structure of the main flow field 
and clearly shows the flowing process to the impeller outlet. At the impeller outlet, there is a significant lowspeed trailing area near the impeller suction surface, which is the typical jet-wake structure [24]. The presence of this structure affects the flow uniformity at the impeller outlet, resulting in the distortion of streamlines. This phenomenon is the most pronounced near the tip clearance in Figure 13(a), while the flow near the hub surface is relatively stable. However, some modes of high-frequency components, such as BPF of $4880 \mathrm{~Hz}$, is shown in Figure 13(b). Unlike the mode of $f_{r}=305 \mathrm{~Hz}$, it is not associated with the mainstream, but reflects the circumferential non-uniformity caused by the impeller rotation. Then, some noteworthy modals appearing in the flow fields are analyzed. The modal with respect to $f=1265 \mathrm{~Hz}$ appears at the impeller outlet but not in the diffuser, while the modal with respect to $f=1175 \mathrm{~Hz}$ appears in the diffuser but not at the impeller outlet, as shown in Figure 13(b). Moreover, these two frequencies seem to have a certain connection. Considering the main blade passing frequency (BPF) is $2240 \mathrm{~Hz}$, the sum of the frequency of $1265 \mathrm{~Hz}$ (51.84\% of main BPF) in the impeller and the frequency of $1175 \mathrm{~Hz}$ (48.16\% of main BPF) in the diffuser happens to be the main BPF. Their pressure modes are shown in Figures 13(c) and (d) respectively. In the impeller mode of $1265 \mathrm{~Hz}$, there are low-pressure zones on the pressure surfaces of the main vanes, resulting in a change of vane loadings. The varying vane loadings affect the strength of the tip leakage flow, leading to a periodic pulsation associated with the main BPF at the impeller outlet. In the diffuser mode of $1175 \mathrm{~Hz}$, due to the above periodic pulsation at the impeller outlet, the high-pressure and low-pressure zones appear alternately in the diffuser. It is related to the mechanism about the circumferential mode generation at the pre-stall condition. In addition, the frequency of the stall inception in the experiment $\left(f_{\text {stall }}=260 \mathrm{~Hz}\right)$ is accidentally found at the diffuser inlet, as shown in Figure 13(e). In the experiment, this frequency occurred gradually during the process from the pre-stall point to the stall inception point, also at the diffuser inlet, as shown in Figure 8. The distribution of this pressure mode in Figure 13(e) looks like a random form of pressure fluctuating circumferentially in the diffuser inlet. It is likely to be the prototype of the stall pattern in Figure 3.

\section{Conclusions}

This paper experimentally and computationally studies the mechanism of the unstable flow structure in an industrial centrifugal compressor with a vaneless diffuser. Through the analysis and discussion, some conclusions are summarized as follows.
(1) In the experiment, a three-lobe stall mode is captured, of which propagating speed is $30.82 \%$ of the impeller rotating speed. Experimental results also demonstrate that due to the asymmetrical distribution of pressure field at the impeller outlet, the prototype of this three-lobe stall mode is gradually formed in the diffuser and its propagation is accelerated with the flow rate reducing. The leakage flow at the impeller middle-rear region multiply the instability of this unstable mode and further trigger a large-scale stall or even surge in the compressor.

(2) In the two-passage numerical simulation of the tested compressor, it can be illustrated that the flow instability at the impeller middle-rear region is co-excited by the tip leakage flow at this region and the flow separation at the leading edge of the impeller main vanes. From the design condition to the pre-stall condition, there are low-speed areas in the tip region at the impeller outlet moving toward the suction surface of the main vanes, resulting in a change of vane loadings. The varying vane loadings affect the strength of the tip leakage flow, leading to a periodic pulsation associated with the main BPF at the impeller outlet.

(3) Through the DMD method, many modes of flow fields related to different frequencies are extracted from the unsteady computational results. One mode with $51.84 \%$ of main BPF is get at the impeller outlet, while another one with $48.16 \%$ of main BPF in the diffuser. Their sum is exactly the main BPF. Owning to the periodic pulsation at the impeller outlet, the corresponding high-pressure and lowpressure zones appeared alternately in the diffuser. These two modes are both related to the flow instability at the pre-stall condition.

Authors' Contributions

TW was in charge of the whole trial; XX did the experiments and laboratory analyses, and wrote the manuscript. Both authors read and approved the final manuscript.

\section{Authors' Information}

Xiang Xue, born in 1992, is currently a PhD candidate at Gas Turbine Research Institute, Shanghai Jiao tong University, China. He received his bachelor degree from Xi'an Jiaotong University, China, in 2014. His research interests include unsteady test and analysis of fluid machineries.

Tong Wang, born in 1969, is currently an associate professor at Gas Turbine Research Institute, Shanghai Jiao tong University, China. She received her PhD degree from Xi'an Jiaotong Universtiy, China, in 1999. Her research interests include performance optimization and numerical calculation of fluid machineries.

Competing Interests

The authors declare that they have no no competing interests. 


\section{Funding}

Supported by National Natural Science Foundation of China (Grant No. 51276108).

Received: 13 April 2018 Revised: 11 March 2019 Accepted: 22 July 2019 Published online: 06 August 2019

\section{References}

[1] I J Day. Stall, surge, and 75 years of research. Journal of Turbomachinery, 2016, 138(1): 1-16.

[2] XXue, TWang, Y C Shao, et al. Experimental and numerical analysis of unsteady flow structure in a centrifugal compressor with variable vaned diffuser. ASME Turbo Expo 2018, Turbomachinery Technical Conference and Exposition, Oslo, Norway, June 11-15, 2018: V02AT45A024

[3] N Fujisawa, T Inui, Y Ohta. Evolution process of diffuser stall in a centrifugal compressor with vaned diffuser. ASME Turbo Expo 2018, Turbomachinery Technical Conference and Exposition, Oslo, Norway, June 11-15, 2018: V02AT45A008.

[4] H J Zhao, Z H Wang, G Xi. Unsteady flow structures in the tip region for a centrifugal compressor impeller before rotating stall. Science China Technological Sciences, 2017: 1-11.

[5] Y P Ju, H Liu, Z Y Yao, et al. Fluid-structure interaction analysis and lifetime estimation of a natural gas pipeline centrifugal compressor under nearchoke and near-surge conditions. Chinese Journal of Mechanical Engineering, 2015, 28(6):1261-1268.

[6] B Semlitsch, M Mihăescu. Flow phenomena leading to surge in a centrifugal compressor. Energy, 2016, 103: 572-587.

[7] X Xue, TWang, TT Zhang, et al. Mechanism of stall and surge in a centrifugal compressor with a variable vaned diffuser. Chinese Journal of Aeronautics, 2018, 31(6): 1122-1231.

[8] S B Ma, KY Kim. Optimization of discrete cavities in a centrifugal compressor to enhance operating stability. Aerospace Science and Technology, 2017, 68: 308-319.

[9] M Gancedo, E Guillou, E Gutmark. Effect of bleed slots on turbocharger centrifugal compressor stability. International Journal of Heat and Fluid Flow, 2018, 133: 464-471.

[10] X F Sun, X Dong, D K Sun. Recent development of casing treatments for aero-engine compressors. Chinese Journal of Aeronautics, 2019, 32(1): $1-36$

[11] X He, X Q Zheng. Roles and mechanisms of casing treatment on different scales of flow instability in high pressure ratio centrifugal compressors. Aerospace Science and Technology, 2019, 84: 734-746.

[12] A Bianchini, D Biliotti, DT Rubino, et al. Experimental analysis of the pressure field inside a vaneless diffuser from rotating stall inception to surge. Journal of Turbomachinery, 2015, 137(11): 111007.

[13] M Marconcini, A Bianchini, M Checcucci, et al. A 3D time-accurate CFD simulation of the flow field inside a vaneless diffuser during rotating stall conditions. ASME Turbo Expo 2016, Turbomachinery Technical Conference and Exposition, Seoul, South Korea, June 13-17, 2016: V02DT42A029.

[14] C Yang, W L Wang, H Z Zhang, et al. Investigation of stall process flow field in transonic centrifugal compressor with volute. Aerospace Science and Technology, 2018, 81: 53-64.
[15] X He, X Q Zheng. Flow instability evolution in high pressure ratio centrifugal compressor with vaned diffuser. Experimental Thermal and Fluid Science, 2018, 98: 719-730.

[16] X J Yang, X C Zhu, C X Hu, et al. Compressed dynamic mode decomposition for the analysis of centrifugal compressor volute. International Journal of Heat and Fluid Flow, 2018, 74: 118-129.

[17] P B Lawless, S Fleeter. Rotating stall acoustic signature in a low-speed centrifugal compressor: Part 1-Vaneless diffuser. Journal of Turbomachinery, 1995, 117(1): 87-96.

[18] P Frigne, RV Braembussche. A theoretical model for rotating stall in the vaneless diffuser of a centrifugal compressor. Journal of Engineering for Gas Turbines and Power, 1985, 107(2): 507-513.

[19] XXue, TWang. Stall recognition for centrifugal compressors during speed transients. Applied Thermal Engineering, 2019, 153:104-112.

[20] S Ohuchida, T Kawakubo, H Tamaki. Experimental study of rotating stall in vaneless diffuser of a centrifugal compressor. ASME Turbo Expo 2013, Turbine Technical Conference and Exposition, San Antonio, Texas, USA, June 3-7, 2013: V06CT40A014

[21] XXue, TWang, J H An. Analysis of modal stall inception in compressors based on phase difference of wavelet transforms. Compressor Blower and Fan Technology, 2017 (3): 20-28.

[22] D Biliotti, A Bianchini, G Vannini, et al. Analysis of the rotordynamic response of a centrifugal compressor subject to aerodynamic loads due to rotating stall. Journal of Turbomachinery, 2015, 137(2): 021002.

[23] X Zheng, A Liu. Phenomenon and mechanism of two-regime-surge in a centrifugal compressor. Journal of Turbomachinery, 2015, 137(8): 081007

[24] M Schleer, R S Abhari. Clearance effects on the evolution of the flow in the vaneless diffuser of a centrifugal compressor at part load condition. Journal of Turbomachinery, 2008, 130(3): 031009.

[25] P G A Cizmas, A Palacios. Proper orthogonal decomposition of turbine rotor-stator interaction. Journal of Propulsion and Power, 2003, 19(2): 268-281.

[26] P J Schmid. Dynamic mode decomposition of numerical and experimental data. Journal of Fluid Mechanics, 2010, 656: 5-28.

[27] E Munari, M Morini, M Pinelli, et al. Experimental investigation of stall and surge in a multistage compressor. Journal of Engineering for Gas Turbines and Power, 2017, 139(2): 022605

[28] J Du, F Lin, J Chen, et al. Flow structures in the tip region for a transonic compressor rotor. Journal of Turbomachinery, 2013, 135(3): 031012.

[29] CW Rowley, I Mezić, S Bagheri, et al. Spectral analysis of nonlinear flows. Journal of Fluid Mechanics, 2009, 641: 115-127.

\section{Submit your manuscript to a SpringerOpen ${ }^{\circ}$ journal and benefit from:}

- Convenient online submission

- Rigorous peer review

- Open access: articles freely available online

- High visibility within the field

Retaining the copyright to your article

Submit your next manuscript at springeropen.com 